\title{
A Reset Kinematic State Estimator to Suppress Sensor Quantization for Enhanced Position Tracking Control
}

\author{
Jinchuan Zheng and Minyue Fu
}

\begin{abstract}
Sensor quantization is one of the key factors that deteriorate the tracking performance of positioning systems with low-resolution optical encoders. This paper presents a reset kinematic state estimator (RKSE) by merging an accelerometer to improve the performance of such systems. The RKSE is immune to both system perturbations and input disturbances and offers more accurate state estimation than the standard state estimator (SSE) without reset. The estimated state is fed back for sinusoidal position tracking control. Experimental results demonstrate the improved tracking accuracy and the robustness with the use of RKSE.
\end{abstract}

\section{INTRODUCTION}

Optical encoders are typically integrated into a linear motor (LM) system to detect the position of a moving object. The encoder is based on evenly spaced divisions or line counts on a glass or metal disk, which is simple in construction and easy to manufacture. However, the interval of the divisions adversely leads to the resolution limitation. When the encoder output is used as the feedback signal in a servo system, the encoder quantization noise will degrade the achievable position accuracy and even cause self-sustained oscillations (i.e., limit cycle) [1].

Quantization is inherently a nonlinear feature. In order to alleviate the sensor quantization effect, numerical algorithms that can be simply implemented on a digital signal processor (DSP) have been reported. For instance, Kalman filters have been employed to suppress the sensor quantization effects under the assumption that quantization effects could be modeled as a white Gaussian noise [2], [3]. However, in motion control systems, signals tend to be more deterministic and exhibit stronger correlation over time. Thus, the quantization noise behaves as highly colored noise, which makes the Kalman filter impractical to employ. In such circumstances, other approaches based on observer theory have been extensively studied in [4] and [5], in which extra useful information is extracted from the quantizer model and then used to enhance the estimation. This paper also presents a solution to this problem along this line by resetting the estimated state based on the encoder output.

In the presence of plant uncertainty, the state estimator becomes unreliable due to the nonlinear quantization noise. A cost-effective way to improve the performance is to incorporate acceleration sensing. There are two main reasons for this option. Firstly, the performance of linear accelerometers have recently improved a lot while the cost and size are

J. Zheng and M. Fu are with the School of Electrical Engineering and Computer Science, The University of Newcastle, Callaghan, NSW 2308, Australia, Jinchuan. Zheng@newcastle.edu.au; Minyue.Fu@newcastle.edu.au

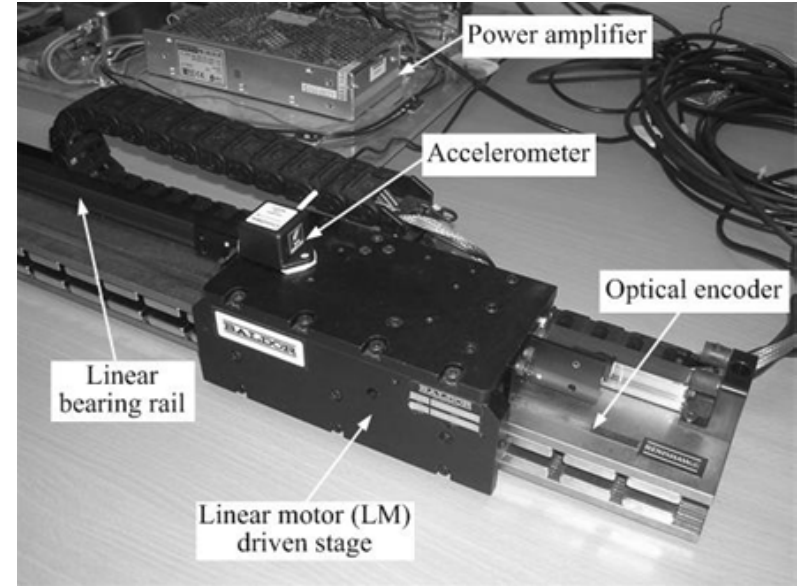

Fig. 1. Experimental setup of an LM positioning system.

both reduced significantly due to mass fabrication and microelectro-mechanical systems (MEMS) technology. Secondly, the use of accelerometers in state estimation can yield a kinematic model from the acceleration to the position, which is independent of the plant parameters [6].

This paper considers an LM positioning system integrated with a low-resolution linear encoder and augmented by an accelerometer. The acceleration signals are used in two ways. One is in the design of a disturbance observer (DOB), the roles of which are for disturbance and friction compensation and for overcoming payload variations. The other is in the design of a reset kinematic state estimator (RKSE). It will be shown that the kinematic state estimator is more or less independent of the system model, thus, is not only robust against plant parameters but also insensitive to the input disturbance and friction force. Moreover, we attempt to further decrease the estimation error by extracting additional information from the quantized output, which is then used to update the estimated state by a reset technique [7]. Finally, we apply the RKSE to a feedback controller and verify its effectiveness by experiments.

\section{Plant Modeling}

The experimental setup for an LM positioning system (by Baldor Electric Company) is shown in Fig. 1. The LM has a $0.5 \mathrm{~m}$ travel range with a mounted optical encoder (by Renishaw PLC), and a power amplifier. A simplified plant model of the LM system is described in Fig. 2. The friction model $g(v)$ can be represented by

$$
g(v)=k_{v f} v+k_{c f} \operatorname{sgn}(v)+k_{s f} \operatorname{sgn}(v) e^{-|v / \sigma|}+\Delta_{f},
$$




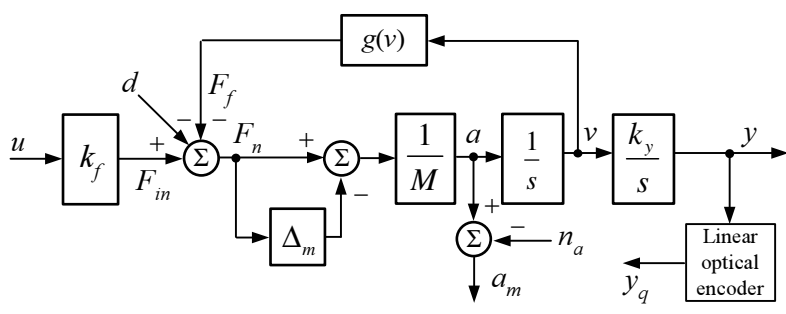

Fig. 2. A plant model of the LM positioning system.

TABLE I

PLANT MODEL PARAMETERS

\begin{tabular}{|c|c|c|c|}
\hline Parameter & Description & Value & Unit \\
\hline$k_{f}$ & voltage-to-force constant & 50 & $N / V$ \\
\hline$k_{y}$ & position sensor gain & $10^{6}$ & $\mu m / m$ \\
\hline$M$ & moving mass of linear motor stage & 3.31 & $\mathrm{~kg}$ \\
\hline$\Delta_{m}$ & gain variation due to load mass & {$\left[\begin{array}{ll}0 & 1\end{array}\right)$} & \\
\hline$\Delta$ & position encoder resolution & 10 & $\mu m$ \\
\hline$\overline{k_{v f}}$ & viscous friction coefficient & 8.6 & $N s / m$ \\
\hline$k_{c f}$ & Coulomb friction level & 11.5 & $N$ \\
\hline$k_{s f}$ & static friction level & 3.3 & $N$ \\
\hline$\sigma$ & static friction velocity constant & 0.023 & $\mathrm{~m} / \mathrm{s}$ \\
\hline
\end{tabular}

where $\operatorname{sgn}(\cdot)$ denotes the sign function, $\Delta_{f}$ represents the unmodeled friction force. Table I lists the LM physical parameters. The friction model is also shown in Fig. 5 later.

The linear optical incremental encoder model is given in Fig. 3, where $n$ represents the encoder imperfections due to optical, mechanical and electrical inaccuracy [8]. Typically, $n$ can be characterized by uniformly distributed noise over $[-\delta,+\delta][9]$. The uniform quantizer is defined by

$$
y_{q}=i \cdot \Delta, \quad \text { if } y_{n} \in[(i-0.5) \Delta,(i+0.5) \Delta]
$$

where $i \in \mathbb{Z}$ and the constant quantization step size $\Delta$ denotes the encoder resolution. We assume that the quantization range is infinite. Apparently, the linear encoder introduces a bounded sensing noise (also referred to as quantization noise in this paper) as follows:

$$
\varepsilon(t)=y(t)-y_{q}(t) .
$$

The physical optical encoder in our setup has a resolution of $1 \mu \mathrm{m}$. But we pass its output through the encoder model in Fig. 3 performed in DSP to simulate a lower resolution encoder with $\Delta=10 \mu \mathrm{m}$ and $\delta=1 \mu \mathrm{m}$. Hence, the high-resolution encoder output is approximated as the actual position $y$ and used for monitoring only, while the artifical low-resolution encoder output $y_{q}$ is used for control purpose.

The linear accelerometer (by Crossbow Inc., see Fig. 1) offers a $\pm 39.2 \mathrm{~m} / \mathrm{s}^{2}$ measurement range with a DC $-100 \mathrm{~Hz}$ frequency bandwidth. Its sensing noise $n_{a}$ is as small as with $R M S=0.027 \mathrm{~m} / \mathrm{s}^{2}$.

\section{Control Structure}

The control task is to make the LM position output follow a sinusoidal reference input under the constraints of friction, load mass variations and the position quantization noise. Fig. 4 shows the overall control structure, where the controller is comprised of three components: the DOB, the

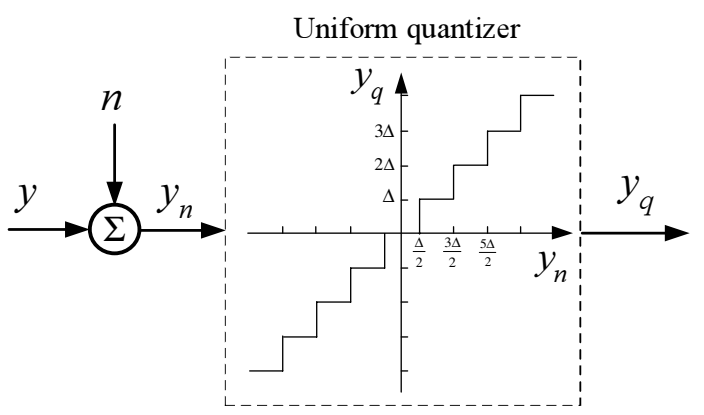

Fig. 3. Model of a linear optical encoder, where $n$ represents encoder imperfections characterized by uniformly distributed noise, $\Delta$ represents encoder resolution.

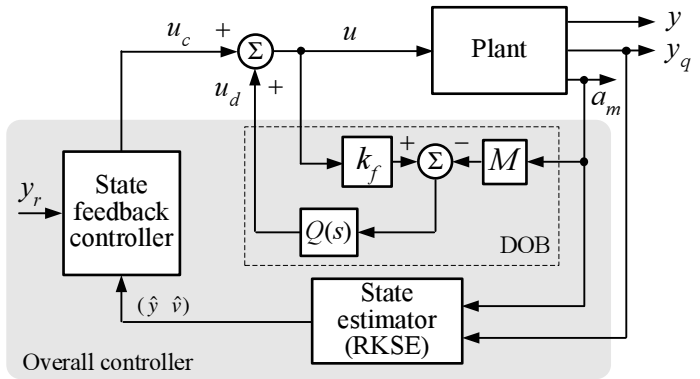

Fig. 4. Overall position control structure for the LM positioning system.

RKSE, and the state feedback controller based on the internal model principle (IMP). Here, the DOB is firstly designed to compensate for external disturbance, friction, and load mass variations. The RKSE aims to suppress the quantization noise and thus to obtain the accurate position and velocity information, which is then used as the input to the state feedback controller for position tracking.

\section{Disturbance ObSERVER (DOB)}

The control structure of the DOB based on acceleration feedback is shown in Fig. 4. The reason for employing acceleration instead of encoder feedback is to avoid introducing the quantization noise into the DOB loop. From Fig. 4 , the sensed signals $u$ and $a_{m}$ go into the DOB and the output signal $u_{d}$ from the low-pass filter $Q(s)$ is fed back to the control input. The dynamics from $u_{c}$ to the actual acceleration $a$ is then given by

$$
\begin{aligned}
a=\frac{1-\Delta_{m}}{1-\Delta_{m} k_{f} Q} & \cdot\left(\frac{k_{f}}{M} \cdot u_{c}+k_{f} Q \cdot n_{a}\right. \\
& \left.+\frac{k_{f} Q-1}{M} \cdot\left(d+F_{f}\right)\right) .
\end{aligned}
$$

Ideally, given that $Q(s) \equiv 1 / k_{f}$, we have

$$
a=\frac{k_{f}}{M} \cdot u_{c}+n_{a}
$$

Thus, the external disturbance and friction force are perfectly canceled, although the measurement noise is all-pass. This again explains the reason for using the acceleration signal as the input instead of the quantized position that contains much a higher level of noise. Practically, $Q(s)$ is selected to be a 


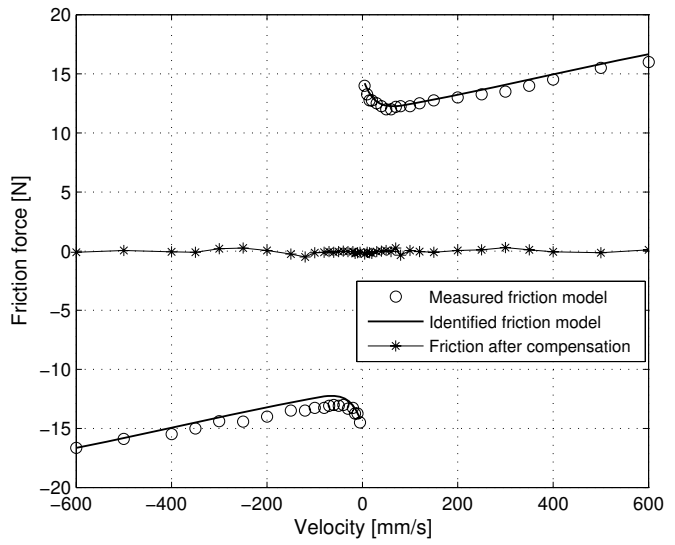

Fig. 5. Experimental friction model.

low-pass filter to balance the compensation of sensing noise and disturbance. Since the nominal model from $u_{c}$ to $a$ can be treated as a constant gain, there is no specific requirement for the order of $Q$ filter. One simple choice of $Q(s)$ can be

$$
Q=\frac{1}{\tau s+k_{f}}
$$

where $\tau$ is a positive time constant chosen as 5 to 10 times the servo bandwidth such that the filter can be approximated as $Q \approx 1 / k_{f}$ within the frequency of interest. When the $Q$ filter is applied, the input-output transfer function of the DOB is given by

$$
\begin{aligned}
a= & \frac{1-\Delta_{m}}{\tau s+k_{f}\left(1-\Delta_{m}\right)}\left(\left(\tau s+k_{f}\right) \frac{k_{f}}{M} \cdot u_{c}\right. \\
& \left.+k_{f} \cdot n_{a}-\frac{\tau s}{M} \cdot\left(d+F_{f}\right)\right) .
\end{aligned}
$$

It is clear that the DOB (6) is stable provided that $\Delta_{m}<1$. Therefore, there exists an $\bar{\omega}>0$ such that

$$
a=\frac{k_{f}}{M} \cdot u_{c}+\omega, \quad|\omega|<\bar{\omega},
$$

where $\omega$ represents an equivalent residual disturbance that is beyond the capability of the DOB to reject. Generally, $\omega$ contains high frequency components, which can be further filtered by the low-pass featured plant model.

Fig. 5 shows the effectiveness of the DOB on friction compensation by experiments. Accordingly, the LM plant model behaves as a second-order linear system given by $P_{n}(s)=\frac{y(s)}{u_{c}(s)}=\frac{k_{f} k_{y}}{M} \cdot \frac{1}{s^{2}}$, which is employed as the nominal model for the design of the state feedback controller in Section VI.

\section{Reset Kinematic State Estimator (RKSE)}

The acceleration and velocity are the two principal quantities which describe how the position changes. A purely kinematic model in the state space that relates the acceleration $a(t)$ to the position $y(t)$ can be presented as follows:

$$
\begin{aligned}
\dot{x}(t) & =A_{r} x(t)+B_{r} a(t), \quad x(0)=x_{0} \\
y(t) & =C_{r} x(t) \\
y_{q}(t) & =C_{r} x(t)-\varepsilon(t) \\
a_{m}(t) & =a(t)-n_{a}(t)
\end{aligned}
$$

where $x=\left[\begin{array}{ll}y & v\end{array}\right]^{T}$ and

$$
A_{r}=\left[\begin{array}{cc}
0 & k_{y} \\
0 & 0
\end{array}\right], B_{r}=\left[\begin{array}{l}
0 \\
1
\end{array}\right], C_{r}=\left[\begin{array}{ll}
1 & 0
\end{array}\right] .
$$

Note that the acceleration is treated as the input, and the system matrices involves none of the plant parameters. Thus, It is clear that the kinematic model has two advantages: it is a simple and exact representation of the system states; while it involves neither physical parameters nor external disturbance, friction, and model uncertainties.

In order to achieve a smooth and accurate estimate of the state using the measured acceleration and the quantized position, the most common way is to use the standard state estimator (SSE) as follows:

$$
\begin{aligned}
\dot{\hat{x}}(t) & =A_{r} \hat{x}(t)+B_{r} a_{m}(t)+L\left(y_{q}(t)-\hat{y}(t)\right) \\
\hat{y}(t) & =C_{r} \hat{x}(t),
\end{aligned}
$$

where $\hat{x}$ and $\hat{y}$ are, respectively, the estimate of the state and controlled output, and $L \in \mathbb{R}^{2 \times 1}$ is the estimator gain. Conventionally, $L$ can be artificially selected by using the pole placement method or optimally designed using the Kalman filter technique. However, we find that more accurate state estimate is possible if the quantization scheme is fully employed.

In Fig. 3, suppose $n$ is very small relative to $\Delta$ and is thus negligible, then we can extract some useful information from the quantized output $y_{q}$ based on two observations: 1) At the time when the quantized output transits from one step to another, the actual position is measured exactly, which locates at the mid-points of the two consecutive quantization levels. 2) At the time when the quantizer holds its output equivalent to a certain quantization step, the actual position relative to the quantized output is always bounded by $\Delta / 2$. This implies that any estimate of $y$ at these times must be bounded by $\Delta / 2$ relative to the instantaneous quantized output. We note that these observations can be used to improve the estimate of the state.

\section{A. Reset Kinematic State Estimator}

For the purpose of estimator design, we first assume that the encoder imperfection $n$ is ignored. Then, it is accounted in simulation and experiment later to reveal its effect on the state estimation.

Firstly, we introduce a constant vector $H \in \mathbb{R}^{2 \times 1}$, which is given by

$$
H=P^{-1} C_{r}^{T}\left(C_{r} P^{-1} C_{r}^{T}\right)^{-1},
$$

where $P \in \mathbb{R}^{2 \times 2}$ is a positive definite symmetric matrix, which is the solution of the following Lyapunov function

$$
\left(A_{r}-L C_{r}\right)^{T} P+P\left(A_{r}-L C_{r}\right)+I=0,
$$

where the estimator gain $L$ is designed such that $A_{r}-L C_{r}$ is stable.

Next, we modify the SSE (10) to incorporate the exacted information from the quantized output. Namely, we reset the estimated state in two cases: 
1) At the reset time $t_{k, 1}$, which is defined as

$$
t_{k, 1}: y_{q}\left(t_{k, 1}\right) \neq y_{q}\left(t_{k, 1}^{-}\right)
$$

the estimated state is reset by

$$
\begin{aligned}
\hat{x}\left(t_{k, 1}\right)= & \hat{x}\left(t_{k, 1}^{-}\right)-H\left(\hat{y}\left(t_{k, 1}^{-}\right)\right. \\
& -\frac{1}{2}\left(y_{q}\left(t_{k, 1}\right)+y_{q}\left(t_{k, 1}^{-}\right)\right) .
\end{aligned}
$$

Then, the new estimated state leads to

$$
\hat{y}\left(t_{k, 1}\right)=\frac{1}{2}\left(y_{q}\left(t_{k, 1}\right)+y_{q}\left(t_{k, 1}^{-}\right)\right)=y\left(t_{k, 1}\right) .
$$

2) At the pre-specified reset time $t_{k, 2}$ defined by

$$
t_{k, 2}=k T, \quad k \in \mathbb{Z}^{+}
$$

where $T$ indicates a predefined reset interval (e.g., sampling period), the estimated state is then reset by

$$
\begin{aligned}
\hat{x}\left(t_{k, 2}\right)= & \hat{x}\left(t_{k, 2}^{-}\right)-H\left(\hat{y}\left(t_{k, 2}^{-}\right)-y_{q}\left(t_{k, 2}\right)-\right. \\
& \left.\operatorname{Sat}_{\frac{\Delta}{2}}\left(\hat{y}\left(t_{k, 2}^{-}\right)-y_{q}\left(t_{k, 2}\right)\right)\right)
\end{aligned}
$$

where $\operatorname{Sat}(\cdot)$ is the saturation function with the saturation level of $\Delta / 2$. We can see that the new estimated state can lead to

$$
\hat{y}\left(t_{k, 2}\right)=\left\{\begin{aligned}
y_{q}\left(t_{k, 2}\right)+\operatorname{Sat}_{\Delta}\left(\hat{y}\left(t_{k, 2}^{-}\right)-y_{q}\left(t_{k, 2}\right)\right), & \text { if }\left|\hat{y}\left(t_{k, 2}^{-}\right)-y_{q}\left(t_{k, 2}\right)\right|>\frac{\Delta}{2} \\
\hat{y}\left(t_{k, 2}^{-}\right), & \text {otherwise. }
\end{aligned}\right.
$$

Hence, it can be seen that over-estimation of $\hat{y}$ is prevented while the estimated output is unchanged if over-estimation is not detected.

By incorporating the preceding reset schemes into the SSE (10), we can obtain a hybrid system named by RKSE, which has the form:

$$
\begin{aligned}
\dot{\hat{x}}(t)= & A_{r} \hat{x}(t)+B_{r} u(t)+L\left(y_{q}(t)-\hat{y}(t)\right), \\
& t \notin\left\{t_{k, 1} ; t_{k, 2}\right\} ; \\
\hat{x}\left(t_{k, 1}\right)= & \hat{x}\left(t_{k, 1}^{-}\right)-H\left(\hat{y}\left(t_{k, 1}^{-}\right)-\frac{1}{2}\left(y_{q}\left(t_{k, 1}\right)+y_{q}\left(t_{k, 1}^{-}\right)\right),\right. \\
& t_{k, 1}: y_{q}\left(t_{k, 1}\right) \neq y_{q}\left(t_{k, 1}^{-}\right) ; \\
\hat{x}\left(t_{k, 2}\right)= & \hat{x}\left(t_{k, 2}^{-}\right)-H\left(\hat{y}\left(t_{k, 2}^{-}\right)-y_{q}\left(t_{k, 2}\right)-\right. \\
& \left.\operatorname{Sat}_{\frac{\Delta}{2}}\left(\hat{y}\left(t_{k, 2}^{-}\right)-y_{q}\left(t_{k, 2}\right)\right)\right), \quad t_{k, 2}=k T ; \\
\hat{y}(t)= & C_{r} \hat{x}(t) .
\end{aligned}
$$

where $\hat{x}\left(t_{k, 1}\right)$ and $\hat{x}\left(t_{k, 2}\right)$ are the new estimated states at time $t_{k, 1}, t_{k, 2}$ respectively.

\section{B. Stability Analysis}

In the following, we analyze the stability of the RKSE (20)-(23). Define the estimator error

$$
e(t) \triangleq x(t)-\hat{x}(t)
$$

Subtracting the estimator (20)-(22) from the kinematic model (8) yields the estimator error system:

$$
\begin{aligned}
\dot{e}(t) & =A_{e} e(t)+B_{r} n_{a}(t)+L \varepsilon(t), e(0)=e_{0}, \\
e\left(t_{k, 1}\right) & =D e\left(t_{k, 1}^{-}\right), \\
e\left(t_{k, 2}\right) & \left.=D e\left(t_{k, 2}^{-}\right)+t_{k, 2}\right\}
\end{aligned}
$$

where

$$
\begin{aligned}
A_{e} & =A_{r}-L C_{r}, D=I-H C_{r} \\
\psi\left(t_{k, 2}\right) & =\varepsilon\left(t_{k, 2}\right)-\operatorname{Sat}_{\frac{\Delta}{2}}\left(\hat{y}\left(t_{k, 2}^{-}\right)-y_{q}\left(t_{k, 2}\right)\right) .
\end{aligned}
$$

We have the following result regarding to the stability of system (25):

Lemma 1: The estimator error system (25) is uniformly bounded-input bounded-state (UBIBS) stable. More specifically, for any $\alpha \geq 0$ and $\bar{n}_{a} \geq 0$, there exists $\mu>0$ such that

$$
|| e(0)\left\|\leq \alpha,\left|n_{a}(t)\right| \leq \bar{n}_{a}, \forall t \geq 0 \Rightarrow\right\| e(t) \| \leq \mu, \forall t \geq 0 .
$$

Proof: The proof can be easily completed following [7].

\section{Simulated Comparison of RKSE and SSE}

We carry out simulation studies on the LM plant model to show the effectiveness of the reset actions in state estimation. The performance index compared is the root mean square (RMS) of the sampled position estimation error $e_{y}$, which is defined by $R M S\left(e_{y}\right)=\sqrt{\sum_{k=1}^{N} e_{y}^{2}(k) / N}$, where $e_{y}=$ $y-\hat{y}$, and $N$ is the number of the samples. The estimator gain $L$ for the RKSE and SSE is chosen as

$$
L=\left[\begin{array}{ll}
4 \pi \zeta f_{n} & 4 \pi^{2} f_{n}^{2} / k_{y}
\end{array}\right]^{T},
$$

where $\zeta=0.707$, and $f_{n}$ is referred to as the estimator bandwidth that is tuned iteratively to achieve the smallest $R M S\left(e_{y}\right)$.

During the simulation, the LM is assumed to rest at an initial position with $y(0)=23 \mu \mathrm{m}$. A simple PID controller with actual position as feedback is designed such that the position output $y$ follows a sinusoidal reference command

$$
y_{r}=r \operatorname{Sin}\left(2 \pi f_{0} t\right)
$$

with $r=50 \mu \mathrm{m}$ and $f_{0}=10 \mathrm{~Hz}$. The linear encoder is with $\Delta=10$ and $\delta=1$ and its output $y_{q}$ together with the acceleration signals added by a white noise with a variance of $7.3 \mathrm{~m} / \mathrm{s}^{2}$ are then input to the RKSE and SSE, respectively. The sampling length is set to be one cycle of the reference, i.e., $0.1 \mathrm{~s}$. The reset interval for RKSE is $50 \mu$ s. The RKSE and SSE are run simultaneously.

Fig. 6 shows the achievable estimation errors versus estimator bandwidth. It is obvious that RKSE can achieve a significant smaller least $R M S\left(e_{y}\right)$ than those by either the SSE or the quantizer. We can also see that a lower bandwidth (equivalent to smaller $L$ ) is allowed for RKSE to alleviate the quantization noise. The corresponding time traces of the position estimation are shown in Fig. 7, which indicates that RKSE has a faster transient convergence to the actual 


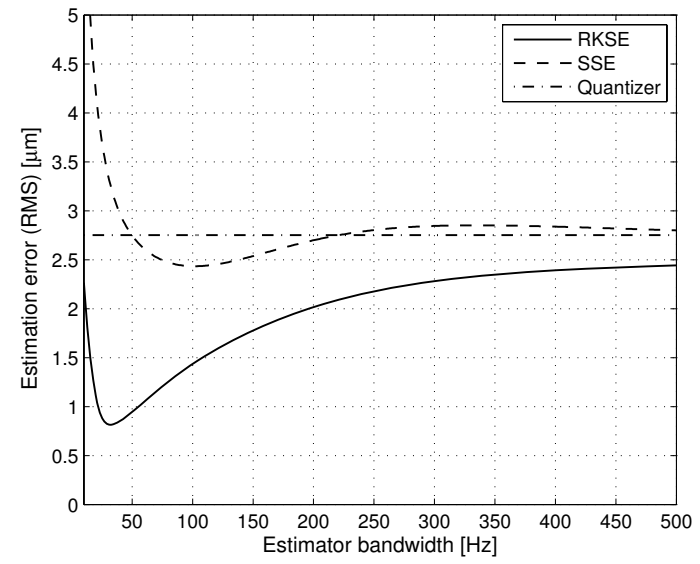

Fig. 6. Position estimation error versus estimator bandwidth.
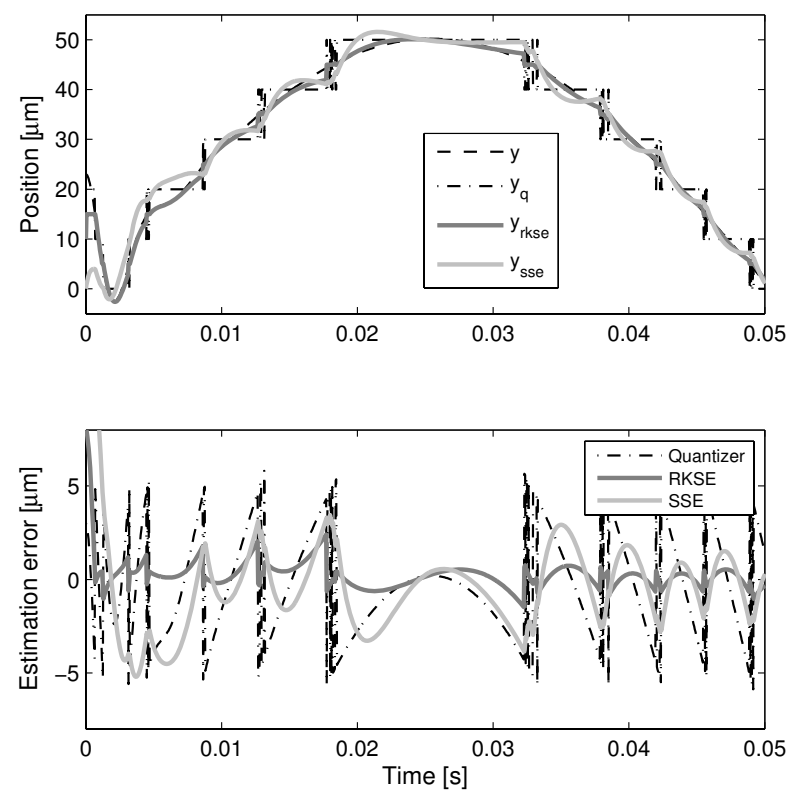

Fig. 7. Time responses of position estimation.

position and a smaller maximal estimation error in steady state. Since the RKSE relies on the edges of the quantized position to reset the estimate, we also study the effect of the encoder imperfections on the state estimation. Fortunately, Fig. 8 shows that the RKSE still outperforms the SSE even with $\delta=40 \% \Delta$, which is beyond the amount of the imperfections in a real linear encoder (typically, $\delta=10 \% \Delta$ ).

\section{State Feedback Control Using IMP}

The state feedback controller using the IMP [10] is designed to make the LM follow a sinusoidal reference command (28) with a known frequency. From Fig. 4, the nominal plant model can be represented in state-space as

$$
\begin{aligned}
& \dot{x}(t)=A x(t)+B u_{c}(t)+\Gamma w(t) \\
& y(t)=C x(t) \\
& \hat{x}(t)=x(t)-e(t)
\end{aligned}
$$

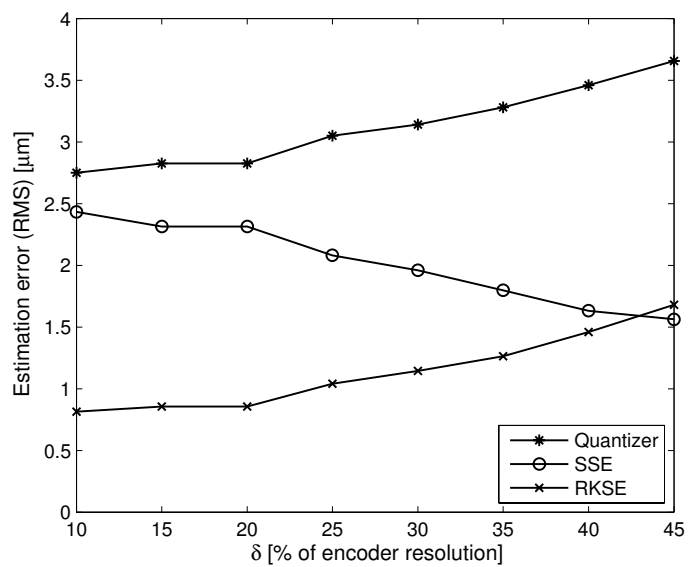

Fig. 8. Position estimation error versus encoder imperfections.

where $x=\left[\begin{array}{ll}y & v\end{array}\right]^{T}$ and

$$
A=\left[\begin{array}{cc}
0 & k_{y} \\
0 & 0
\end{array}\right], B=\left[\begin{array}{c}
0 \\
\frac{k_{f}}{M}
\end{array}\right], \Gamma=\left[\begin{array}{l}
0 \\
1
\end{array}\right], C=\left[\begin{array}{ll}
1 & 0
\end{array}\right] .
$$

Note that $\omega$ is the residual disturbance from the DOB as shown in (7), and $e$ is the estimation error (24).

For the purpose of control design, we first assume that the full state feedback is available and $w(t)$ is ignored, which, however, are accounted in system analysis later. For the reference input (28), we then have the differential equation

$$
\ddot{y}_{r}+\omega_{0}^{2} y_{r}=0 .
$$

Define the tracking error as $e_{t}=y_{r}-y$, and a new state and control input as follows:

$$
\begin{aligned}
& \xi \triangleq \ddot{x}+\omega_{0}^{2} x, \\
& \mu \triangleq \ddot{u}_{c}+\omega_{0}^{2} u_{c},
\end{aligned}
$$

which relate the original plant state and control input to the differential equation of the reference (31). Accordingly, an overall system state equation can be described as

$$
\begin{aligned}
\dot{z} & =\left[\begin{array}{cc|c}
0 & 1 & 0_{1 \times 2} \\
-w_{0}^{2} & 0 & -C \\
\hline 0_{2 \times 1} & 0_{2 \times 1} & A
\end{array}\right] z+\left[\begin{array}{c}
0_{2 \times 1} \\
\hline B
\end{array}\right] \mu \\
& \triangleq F z+G \mu,
\end{aligned}
$$

where $z \triangleq\left[\begin{array}{lll}e_{t} & \dot{e}_{t} & \xi\end{array}\right]^{T}$. It is easy to verify that $(F, G)$ is controllable, which implies that the error system (34) can have arbitrary dynamics by state feedback. Therefore, there exists a control law

$$
\mu=-\left[\begin{array}{llll}
k_{1} & k_{2} & k_{3} & k_{4}
\end{array}\right] z \triangleq-K z,
$$

such that the error system (34) has arbitrary dynamics by pole placement. Expressing the control law in terms of $u_{c}$ and $x$ gives that

$$
u_{c}=-\frac{k_{2} s+k_{1}}{s^{2}+\omega_{0}^{2}} \cdot e-\left[\begin{array}{ll}
k_{3} & k_{4}
\end{array}\right] x .
$$

The actual state is unavailable in practice, which should be replaced by the estimated state from either the SSE or RKSE. 

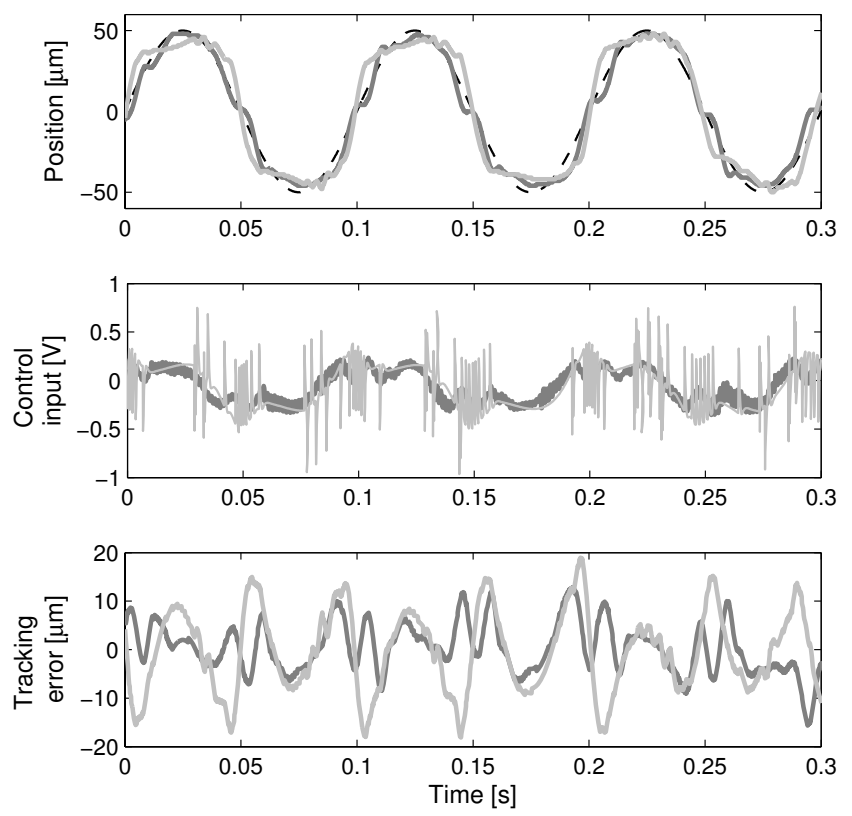

Fig. 9. Experimental position tracking performance (Dark gray lines: with acc and RKSE; Light gray lines: without acc and with SSE, dashed lines: reference).

\section{EXPERIMENTAL RESULTS}

Experiments are conducted on the LM positioning system to demonstrate the advantages of the DOB and RKSE with accelerometer and compare with the conventional DOB and SSE without accelerometer. In the experiments, the same state feedback controller is used in all cases. The state feedback gain $K$ in (35) is designed such that the bandwidth of the overall closed-loop system is $20 \mathrm{~Hz}$. For the control scheme using accelerometer, the bandwidth of the $Q$ filter (5) in the DOB is chosen as $600 \mathrm{~Hz}$, while the bandwidth of RKSE is chosen to minimize the RMS of tracking error. The linear encoder is again with $\Delta=10$ and $\delta=1$. For the control scheme without accelerometer, the DOB is replaced by our previous design in [11] that uses the quantized position signals as input. Further, the SSE uses the control signals $u_{c}$ and quantized position as inputs and its bandwidth is chosen to minimize the RMS of tracking error. Interestingly, we find that the bandwidth of SSE that achieves the smallest RMS of estimation error does not always lead to the smallest RMS of tracking error. This is mainly because the quantization noise in the case of sinusoidal position tracking is nonstationary and harmonic.

The overall controller was discretized and implemented on a real-time DSP system (dSPACE-DS1103, dSPACE GmbH, Paderborn, Germany) with the sampling period $0.2 \mathrm{~ms}$. Fig. 9 shows the position tracking result, which indicates that the tracking error with acc and RKSE is much smaller than that without acc and with SSE. Moreover, the middle plot indicates that the control input with RKSE is smoother and has less signal chattering than that with SSE. We believe that the chattering is mainly caused by the quantization noise that enters the DOB without using accelerometer. This is
TABLE II

EXPERIMENTAL ROBUST TRACKING PERFORMANCE

\begin{tabular}{c|c|c|c|c}
\hline Performance & \multicolumn{2}{|c|}{ with acc and RKSE } & \multicolumn{2}{c}{ without acc and with SSE } \\
\cline { 2 - 5 } index & w/o load & w/ load & w/o load & w/ load \\
\hline $\operatorname{RMS}\left(e_{t}\right)$ & 6.00 & 5.62 & 8.13 & 7.25 \\
$\max \left(\left|e_{t}\right|\right)$ & 15.52 & 16.79 & 18.97 & 15.97 \\
\hline
\end{tabular}

because the chattering occurs at the points where the rate of change of the position is fast, which implies the corresponding quantization noise features frequent zero crossings at these points. Finally, we evaluated the performance with a $1 \mathrm{~kg}$ payload. The results are shown in Tab. II, which indicates that the controller with accelerometer and RKSE has better robustness in terms of smaller difference between with payload and no payload. This verifies the effectiveness of the DOB on the compensation for gain variations.

\section{CONCLUSIONS}

This paper has attempted to enhance the tracking performance of an LM control system by incorporating a costeffective accelerometer. The controller is composed of the DOB, the RKSE, and the IMP-based feedback controller, which are sequentially designed based on the fusion of the acceleration and quantized position signals. Particularly, we apply the reset technique to the RKSE to enhance the suppression of the quantization noise. Simulated and experimental results have demonstrated that the use of accelerometer and RKSE can significantly enhance the position tracking performance and maintain the robustness to system gain variations caused by payload.

\section{REFERENCES}

[1] J. Bertram, "The effect of quantization in sampled-feedback systems," Trans. AIEE, vol. 77, pt. 2, pp. 177-182, Sep. 1958.

[2] D. Luong-Van, M. Tordon, and J. Katupitiya, "Covariance profiling for an adaptive Kalman filter to suppress sensor quantization effects," in Proc. IEEE Conf. Decision and Control, 2004, pp. 2680-2685.

[3] S. Chang and M. Perng, "State estimation from incremental sensor data corrupted by track miscounts and a detection delay," IEEE Trans. Contr. Syst. Technol., vol. 4, no. 1, pp. 65-71, Jan. 1996.

[4] J. Sur and B. Paden, "State observer for linear time-invariant systems with quantized output," Journal of Dynamic Systems, Measurement, and Control, vol. 120, pp. 423-426, Sep. 1998.

[5] D. Delchamps, "Extracting state information from a quantized output record," System and Control Letters, vol. 13, pp. 365-372, 1989.

[6] S. Jeon and M. Tomizuka, "Benefits of acceleration measurement in velocity estimation and motion control," Control Engineering Practice, vol. 15 , pp. 325-332, 2007.

[7] J. Zheng and M. Fu, "A reset state estimator for linear systems to suppress sensor quantization effects," in Proc. 17th IFAC World Congress, 2008, pp. 9254-9259.

[8] C. Yien, "Incremental encoder error: Causes and ways to reduce them," in Proc. Int. Incremental Motion Conf., 1992, pp. 110-121.

[9] R. Kavanagh, and J. Murphy, "The effects of quantization noise and sensor nonideality on digital differentiator-based rate measurement," IEEE Trans. Instrumentation and Measurement, vol. 47, no. 6, pp. 1457-1463, Dec. 1998.

[10] F. Lewis, Applied Optimal Control and Estimation, Digital Design and Implementation, Prentice Hall, New Jersey, 1992.

[11] J. Zheng and M. Fu, "Nonlinear feedback control of a dual-stage actuator system for reduced settling time," IEEE Trans. Contr. Syst. Technol., 2008. vol. 16, no. 4, pp. 717-725, Jul. 2008. 